\title{
Gas Embolism Caused by Portal Vein Gas: Case Report and Literature Review
}

\author{
Chiaki Kamikado $^{a}$ Shinjiro Nagano ${ }^{b}$ Kouji Takumic \\ Terutoshi Senokuchi $^{c}$ Masaaki Kubo ${ }^{a}$ Shinji Mitsue ${ }^{a}$ \\ Toshitaka Fukumoto $^{\text {a }}$ Shouji Natugoe $^{d}$ Takashi Aikou $^{d}$ \\ Departments of aSurgery and ${ }^{\mathrm{b}} \mathrm{Cardiology}$, Kagoshima Prefectural Sastunan \\ Hospital, Minamisatuma City, and Departments of 'Radiology and \\ ${ }^{d}$ Gastrointestinal Surgery, Surgical Oncology, Kagoshima University School of \\ Medicine, Kagoshima City, Japan
}

\section{Key Words}

Portal vein gas - Gas embolism - Portal hypertension - Pulmonary hypertension .

Pulmonary edema

\begin{abstract}
Introduction: We describe a case of pulmonary gas embolism caused by portal vein gas (PVG) observed using echocardiography. Echography revealed gas flowing through the hepatic vein, inferior vena cava, right atrium, and right ventricle, as well as pulmonary hypertension. The patient was diagnosed as having pulmonary gas embolism caused by PVG.
\end{abstract}

Objective: We consider PVG routes to pulmonary circulation, diagnosis of gas embolism caused by PVG, and treatment of gas embolism caused by PVG.

Methods: We reviewed reports of eight cases of gas embolism caused by PVG and compared these cases to cases of gas embolism without PVG.

Results: Mortality of gas embolism caused by PVG was $67 \%$, positive blood culture was observed in six cases, and pulmonary edema was seen in three cases. PVG initially excites microbubble formation, which causes tissue damage in the liver and liver abscess. A large volume of PVG causes portal obstruction. As a result, portal hypertension, a portosystemic shunt or gastrointestinal congestion can occur. PVG can travel to the systemic vein through the liver or portosystemic shunt without anomaly and cause pulmonary gas embolism, followed by arterial embolism. In this environment, sepsis easily occurs. Echocardiography is useful for diagnosis of gas embolism caused by PVG, but the gas can be seen intermittently. The view of pulmonary edema is important for pulmonary gas embolism caused by PVG.

Conclusion: It is important to treat the underlying disease, but PVG must be considered and treated as the gas embolism's source. 


\section{Introduction}

Pulmonary gas embolism is the entry of gas into the venous system because of trauma, iatrogenic complications, laparoscopic procedures, neurosurgical procedures or decompression sickness [1]. A large volume of gas causes mechanical obstruction of the pulmonary artery, pulmonary arterial hypertension, increased resistance to the right ventricle, diminished venous return, and diminished cardiac output [2]. Barak and Katz have summarized the process: when microbubbles obstruct blood flow in the capillary, mechanical factor, inflammatory response, complement activation and clotting activation together facilitate an increase in membrane permeability (pulmonary edema) and endothelial damage (tissue damage) in the lungs [3]. A few microbubbles can coalesce into one large bubble. This obstruction causes an increase in alveolar dead space and intrapulmonary right-to-left shunting. Some of the gas slowly dissolves in the blood, while some excites the lungs, and some travels into the arterial circulation through the lung [4] or patent foramen ovale [5]. When there is a right-to-left shunt, venous air emboli may travel into arterial circulation and cause organ ischemia. Such a course of events is termed paradoxical air embolism [3], and it can cause systemic artery embolism. A patent foramen ovale is detectable in about $30 \%$ of the general population [6]. Obstruction of either the coronary arteries or the nutritive arteries of the brain is especially serious [7]. The patient's comorbidity may also influence the outcome of circulating air emboli.

Portal vein gas (PVG) was first described in children by Wolfe and Evans [8] in 1955 and in adults by Susman and Senturia [9] in 1960. Gas in the hepatic portal venous system can be due to the presence of gas under pressure in the bowel lumen, or by alteration of the bowel mucosa that allows gas to enter the portal system through the mesenteric vein [10]. A large number of cases have been reported in the literature, providing information about the prognosis, causes, mortality, and treatment of PVG. However, it is not well known that PVG can cause pulmonary and systemic gas embolism.

\section{Case Report}

The patient was a 94-year-old woman with senile dementia who consulted a local physician because her temperature was $38^{\circ} \mathrm{C}$. Antibiotics were prescribed. Two days later, after experiencing symptoms of acute dyspnea, vomiting, and fresh melena, the patient was admitted by the physician. She was admitted to our hospital $2 \mathrm{~h}$ later.

At the time of admission, the patient's abdomen was distended but soft. Her body temperature was $35.7^{\circ} \mathrm{C}$. Rales were auscultated in the right lung, and the jugular vein was distended. Hematological and biochemical examination showed no leukocytosis (WBCs 5,190/ $\mu$ l). The CRP level was slightly increased $(1.84 \mathrm{mg} / \mathrm{dl})$, and slight anemia was noted $(\mathrm{Hb} 10.1 \mathrm{~g} / \mathrm{dl})$. Minimal coagulation problems were noted. Renal function was poor (BUN $52.2 \mathrm{mg} / \mathrm{dl}$; Cr $2.0 \mathrm{mg} / \mathrm{dl}$; base excess $-9.9 \mathrm{mEq} / \mathrm{l}$ ) because of dehydration. Liver function studies (e.g. AST, ALT, and LDH) were normal. Arterial blood gas analysis showed $\mathrm{PO}_{2}$ of $56.9 \mathrm{~mm} \mathrm{Hg}$ and $\mathrm{PCO}_{2}$ of $28.2 \mathrm{~mm} \mathrm{Hg}$, with an oxygen flow rate of $5 \mathrm{l} / \mathrm{min}$.

An abdominal X-ray obtained on admission showed dilation of the colon. Abdominal ultrasonography revealed microparticles along with strongly echogenic particles that flowed in the intrahepatic portal and hepatic veins (fig. 1a, video 1a, www.karger.com/doi/10.1159/000146064). An abdominal plain CT obtained on admission showed intrahepatic portal gas (fig. $2 \mathrm{~g}$ ), splenic vein gas (fig. 2h), superior mesenteric vein gas (fig. 2i), and dilation of the colon, but no evidence of bowel wall thickening or ascites. The patient was diagnosed with PVG. Echocardiography showed strong microparticles - strongly echogenic particles that flowed in the right atrium and right ventricle (fig. 1b, video 1b, www.karger.com/doi/10.1159/000146064) - and revealed pulmonary hypertension (PAP $61.8 \mathrm{~mm} \mathrm{Hg}$ ). The strongly echogenic particles were identified as gas passing through the liver, and we diagnosed the patient as having pulmonary gas embolism caused by PVG. 
A comparison of the chest X-ray obtained on admission with the one obtained $2 \mathrm{~h}$ before admission showed rapid spread of infiltration (fig. 2a, b). The chest CT scan performed on admission had shown pulmonary edema located mainly on the ventral side of the right upper lobe of the lung, along the bronchus (fig. 2e). The views on CT were heterogeneous, because the gas floated in liquid and the patient usually lay in the supine position, and in that position, the right pulmonary artery was anatomically higher than the left pulmonary artery.

The patient's family did not agree to a laparotomy, and based on abdominal and biochemical findings, it was decided to follow the patient conservatively. The following treatment plan was implemented: fasting for 5 days; purgatives and antibiotics for 3 days; $2 \mu \mathrm{g} / \mathrm{kg} / \mathrm{min}$ of dopamine for 4 days; and oxygen for 10 days. At night on day 1 of hospitalization, the patient passed a huge mass of stools.

On day 2, a follow-up of abdominal X-ray obtained showed a decrease in the volume of gas in the colon. Abdominal ultrasonography performed that day did not reveal portal gas but showed ascites and bowel wall thickening. Echocardiography performed did not show gas, but revealed PAP of $35.0 \mathrm{~mm} \mathrm{Hg}$. Decreasing the oxygen flow rate resulted in a drop in pulse oximetry readings to less than $90 \%$ of $\mathrm{SPO}_{2}$, so we continued supplemental oxygen.

Cultures of sputum, blood, and stool obtained on the day of admission did not show pathogenicity. Throughout the course of hospitalization, the results of her liver function tests were good, WBC count was normal, and CRP was less than $4.37 \mathrm{mg} / \mathrm{dl}$. By day 2 , her renal function had recovered.

On day 3, a follow-up chest X-ray showed a hydrothorax and no decrease in infiltration (edema) of the lung (fig. 2c). On day 5 , the patient was given a meal. On day 6 , arterial blood gas analysis showed $\mathrm{PO}_{2}$ of $71.7 \mathrm{~mm} \mathrm{Hg}$ and $\mathrm{PCO}_{2}$ of $56.1 \mathrm{~mm} \mathrm{Hg}$, with an oxygen flow rate of $3 \mathrm{l} / \mathrm{min}$. Hypoxia and hypercapnia persisted for at least 10 days after admission. Therefore intrapulmonary right-to-left shunting might play a role in the PAP decrease.

On day 13, an enhanced abdominal CT scan showed that both ascites and bowel wall thickening had disappeared. A chest CT scan obtained the same day showed decreased edema (fig. 2f). A chest X-ray on day 16 showed no signs of hydrothorax (fig. 2d). On day 16, an upper gastrointestinal endoscopy did not find any specific abnormalities. On day 17, a colonoscopy did not find any specific abnormalities. Perhaps alteration of the colon mucosa was healing at the time of examination. The patient was discharged 20 days after hospitalization.

\section{Effect on Portal Circulation}

Mallens et al. have hypothesized that gas can cause obstruction of the portal sinusoids, leading to gas in the inferior mesenteric vein, superior hemorrhoidal vein, and internal iliac veins as can be seen on the CT scan [11]. Studies in dogs injected with air in the portal vein have shown that PVG causes portal hypertension and that hypertension depends on the volume of air [12]. In our case, bowel wall thickness and ascites were observed. A large volume of PVG obstructed the intrahepatic portal vein and increased presinusoidal vascular resistance, leading to portal hypertension, gastrointestinal congestion, gastrointestinal edema, ascites, and development of a portosystemic shunt.

\section{Routes to the Systemic Vein}

In adults, PVG can pass to the systemic vein via two routes: through the liver [13] or through a portosystemic shunt [11]. Studies in dogs have shown that microbubbles can be released into the inferior vena cava via the hepatic vein [14]. In our case, hepatic vein gas followed the route through the liver. In a case report of an infant with necrotizing enterocolitis, the route was through a patent duct [15]. Another route through an enterovenous fistula caused by a tumor or polysurgery is also suspected [13]. 


\section{Effect on Pulmonary and Other Organs}

If a person does not have any anatomical anomaly, PVG will travel through the systemic venous system. Pulmonary gas embolism is the entry of gas into the venous system. Some occurrences of pulmonary gas embolism can cause systemic artery embolism. Therefore, PVG causes pulmonary gas embolism and arterial gas embolism. Five previous cases of adults with systemic vein gas associated with PVG (pulmonary gas embolism caused by PVG) have previously been reported (table 1) $[11,13,16]$. In addition, three cases of systemic arterial gas associated with PVG (arterial gas embolism caused by PVG) have been reported in adults (table 2) [12, 17, 18]. In these previous cases, pulmonary arterial pressure, central venous pressure, and blood gas were not reported. In our patient, we observed pulmonary hypertension, pulmonary edema, right-to-left shunting, hypoxia, and hypercapnia. We suggest that hypotensive shock without sepsis in one case was right heart failure caused by pulmonary gas embolism [11].

In five cases, blood cultures were positive, while two cases presented patent foramen ovale, three cases pulmonary edema, and one case laparoscopy. The causes were not characteristic. The mortality of pulmonary gas embolism caused by PVG was $50 \%$. The mortality of arterial gas embolism caused by PVG was $100 \%$. The overall mortality of PVG patients $(\mathrm{n}=182)$ was $39 \%$ [19]. It is not easily possible to compare these mortalities, but it is clear that it is important to prevent PVG from flowing into other circulatory systems.

\section{Does PVG Excite Ventilation?}

PVG has been reported to have a high $\mathrm{CO}_{2}$ concentration [16], and $\mathrm{CO}_{2}$ is a highly soluble gas. The high solubility of PVG may easily excite ventilation by stimulating receptors within the lungs during expiration, but little is currently known about this component of PVG.

\section{Microbubbles}

PVG is caused by alteration of gastrointestinal mucosa and injury of the large vein is not required. Echography has revealed microbubbles in PVG patients, and thus we hypothesize that PVG initially exists in a microbubble state. Microbubbles are caused by an obstruction of blood flow in the sinusoid or presinusoid vessels. Microbubbles in a low-flow environment float and fuse to create larger bubbles. As in the lung, these microbubbles cause tissue damage in the liver; therefore, as the sinusoidal (microvascular) compliance decreases, the diameter mechanically enlarges, and the microbubbles overflow. Some of the large bubbles divide the microbubbles in the sinusoid, and the overflow gases flow through the heart into the pulmonary circulatory system and cause pulmonary gas embolism. In the environment where microbubbles travel to the venous circulation, bacteria can also travel to the venous circulation. Therefore, in systemic vein gas or systemic arterial gas associated with PVG, the frequency of positive blood culture is high. We regard liver abscess [20] associated with PVG as a result of tissue damage in the liver. 


\section{Diagnosis of Pulmonary Gas Embolism}

Echocardiography is useful for measuring pulmonary artery pressure, diagnosing cardiac defects, and detecting microbubbles. Abdominal and pelvic CT scans are useful for detecting large bubbles of venous gas, while chest X-rays and chest CT scans are useful for detecting pulmonary edema. The gas is only visible intermittently, so pulmonary edema caused by PVG is often diagnosed as pulmonary gas embolism without detecting systemic venous gas. The pathologic picture in pulmonary gas embolism is variable and ranges from mild interstitial edema to hemorrhagic airspace consolidations [21]. A Swan-Ganz catheter is useful for measuring pulmonary arterial pressure and ruling out left-side heart failure. Blood gas is useful, because pulmonary gas embolism can cause hypoxia followed by intrapulmonary or paradoxical right-to-left shunt, causing both hypoxia and hypercapnia.

\section{Diagnosis of Arterial Embolism}

Similarly, it is difficult to detect arterial gas associated with PVG. Neurogenic symptoms must be carefully observed. ST elevation on EKG or infarction view is an important diagnostic indicator in cardiac cases.

\section{Treatment}

The most important treatments are those that prevent the spread of gas. Treatment of the underlying condition and drainage of gastrointestinal gas must be undertaken immediately. Emergent surgery in bowel necrosis cases and drainage (e.g. long tube, stoma, purgative) must also be performed as soon as possible. Volume expansion increases central venous pressure, blocking the systemic vein where PVG flows. In pulmonary embolism or right heart failure, the associated PVG central venous pressure is high, and a Swan-Ganz catheter can be useful for administering fluids or catecholamine.

Treating gas embolism requires volume expansion, a high concentration of oxygen, evacuation of gas from the right ventricle, hyperbaric oxygen, and drugs (e.g. heparin, barbiturates, corticosteroids or lidocaine). A high concentration of oxygen is useful for hypoxia and excites the nitrogen in the gas bubbles $[1,3]$. In gas embolism caused by PVG, such treatments must be undertaken as well.

There are three differences between gas embolism caused by PVG and iatrogenic gas embolism: (1) The majority of underlying diseases in PVG are from septic sources.

(2) Treating some cases of PVG require surgery. (3) PVG consists of microbubbles. The component is not air without endoscopic case. The component is unknown but includes high $\mathrm{CO}_{2}$.

A blood culture must be collected, if possible from the right atrium. If surgery is done, the blood of the mesenteric vein or excised materials should also undergo bacterial culture.

Surgical matters that require attention include: (1) halothane or isoflurane anesthesia may raise the threshold for a spillover of venous bubbles into systemic arteries, and (2) the use of positive end-expiratory pressure has been advocated to prevent and treat pulmonary gas embolism [22], but it may increase the probability of paradoxical air embolism [23] and cardiovascular depression [24]. 
In PVG, microbubbles and high concentration $\mathrm{CO}_{2}$ bubbles may be suitable for hyperbaric oxygen therapy.

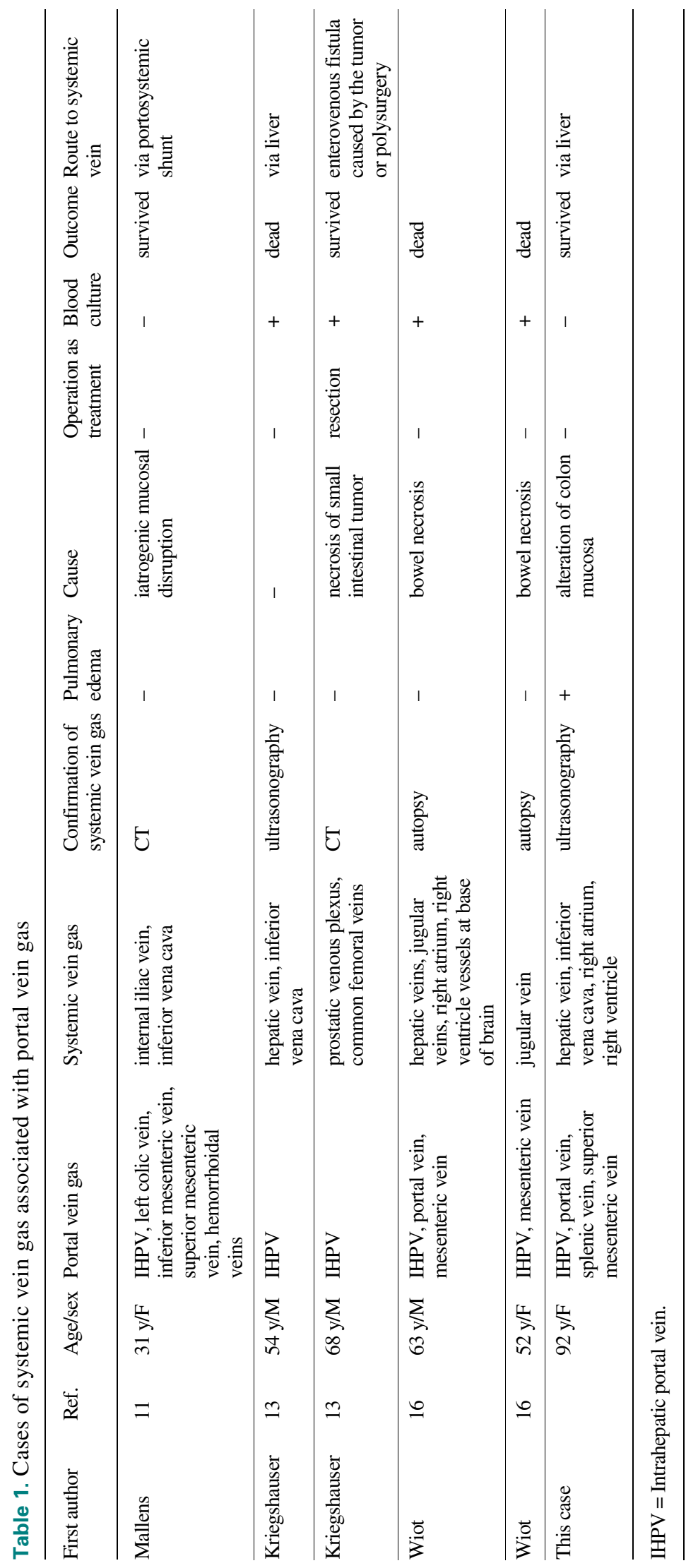


Table 2. Cases of systemic arterial embolisms associated with portal vein gas

\begin{tabular}{llllllllll}
\hline $\begin{array}{l}\text { First } \\
\text { author }\end{array}$ & Ref. & $\begin{array}{l}\text { Age/ } \\
\text { sex }\end{array}$ & $\begin{array}{l}\text { Systemic arterial } \\
\text { gas }\end{array}$ & $\begin{array}{l}\text { Confirmation of } \\
\text { systemic arterial gas }\end{array}$ & $\begin{array}{l}\text { Pulmonary } \\
\text { edema }\end{array}$ & $\begin{array}{l}\text { Cause } \\
\text { Roperation }\end{array}$ & $\begin{array}{l}\text { Blood } \\
\text { culture } \\
\text { treatment }\end{array}$ & $\begin{array}{l}\text { Out- } \\
\text { come }\end{array}$ & $\begin{array}{l}\text { Patent } \\
\text { foramen } \\
\text { ovale }\end{array}$ \\
\hline Root & 12 & $19 \mathrm{y} / \mathrm{F}$ & $\begin{array}{l}\text { aorta, coronary } \\
\text { artery }\end{array}$ & autopsy & + & $\begin{array}{l}\text { laparos- } \\
\text { copy }\end{array}$ & - & - & dead \\
\hline Jones & 17 & $71 \mathrm{y} / \mathrm{F}$ & $\begin{array}{l}\text { iliac artery, femoral } \\
\text { artery }\end{array}$ & X-ray & + & - & - & + & dead \\
\hline Etsuda & 18 & $61 \mathrm{y} / \mathrm{M}$ & $\begin{array}{l}\text { coronary artery } \\
\text { cardiac } \\
\text { catheterization }\end{array}$ & - & $\begin{array}{l}\text { bowel } \\
\text { necrosis }\end{array}$ & resection & - & dead + \\
\hline
\end{tabular}

Fig. 1. Abdominal ultrasonography and echocardiography. a Abdominal ultrasonography on the first day of hospitalization revealed strong and micro echogenic particles. The particles, composed of gas, flowed downstream in the intrahepatic portal vein (black arrows) and downstream or upstream in the hepatic vein (white arrows). Arrowheads indicate the inferior vena cava. Video la (www.karger.com/doi/10.1159/000146064). b Echocardiography revealed strongly linear and micro echogenic particles of various lengths within the right atrium (RA) and right ventricle (RV). The particles were gas (arrows). Video lb (www.karger.com/doi/10.1159/000146064).
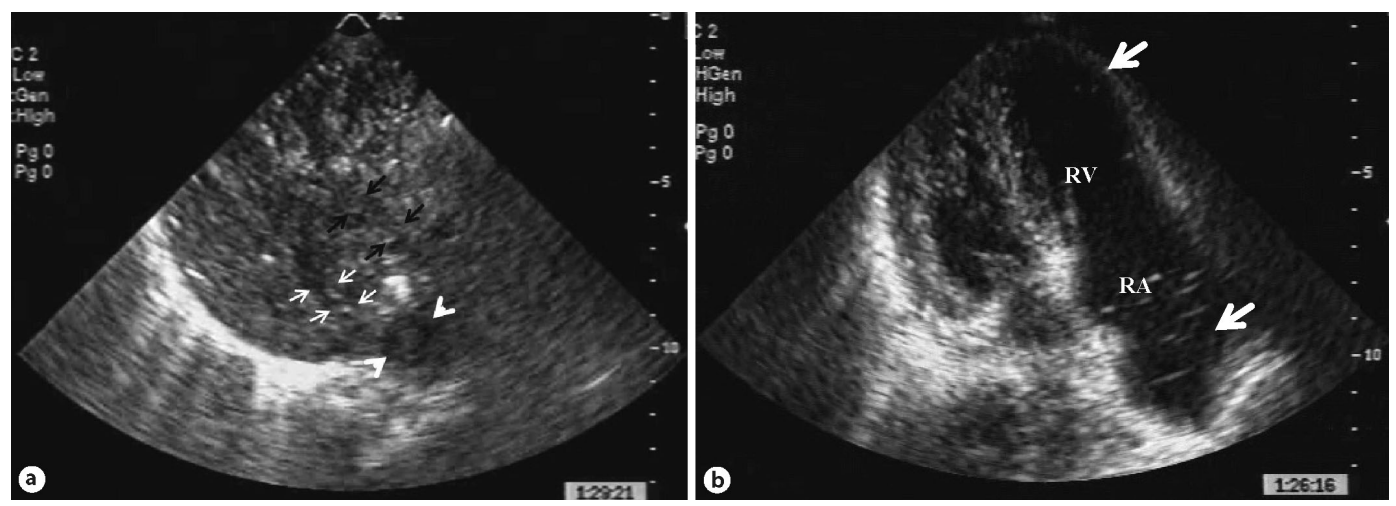


\begin{tabular}{r|l|l|l}
$\begin{array}{r}\text { Case Reports in } \\
\text { Gastroenterology }\end{array}$ & $\begin{array}{l}\text { Case Rep Gastroenterol 2008;2:262-271 } \\
\text { D0I: 10.1159/000146064 }\end{array}$ & Published online: August 15, 2008 & $\begin{array}{l}\text { O 2008 S. Karger AG, Basel } \\
\text { ISSN 1662-0631 } \\
\text { www.karger.com/crg }\end{array}$ \\
\hline
\end{tabular}

Fig. 2. Chest X-ray and CT. a Chest X-ray at 11:00 am on day 1 of hospitalization, obtained $2 \mathrm{~h}$ before admission, shows infiltration mainly in the right upper lung field. b Chest X-ray at 1:00 p.m. on day 1 shows extensive spread of infiltration when compared with $\mathbf{a}$. c Chest X-ray on day 3 shows bilateral pulmonary effusion and infiltration. $\mathbf{d}$ Chest X-ray on day 16 shows the disappearance of effusion and infiltration. e Plain CT of the chest on admission shows pulmonary edema located mainly on the ventral side of the right lobe of the lung along the bronchus. $\mathbf{f}$ Enhanced CT scan of the chest on day 13 shows bilateral pulmonary effusion and decreased pulmonary edema. $\mathbf{g}$ Plain CT scan of the abdomen on admission shows intrahepatic portal gas. $\mathbf{h}$ Plain CT scan of the abdomen on admission shows splenic vein gas (arrow). i Plain CT scan of the abdomen on admission shows superior mesenteric vein gas (arrow).
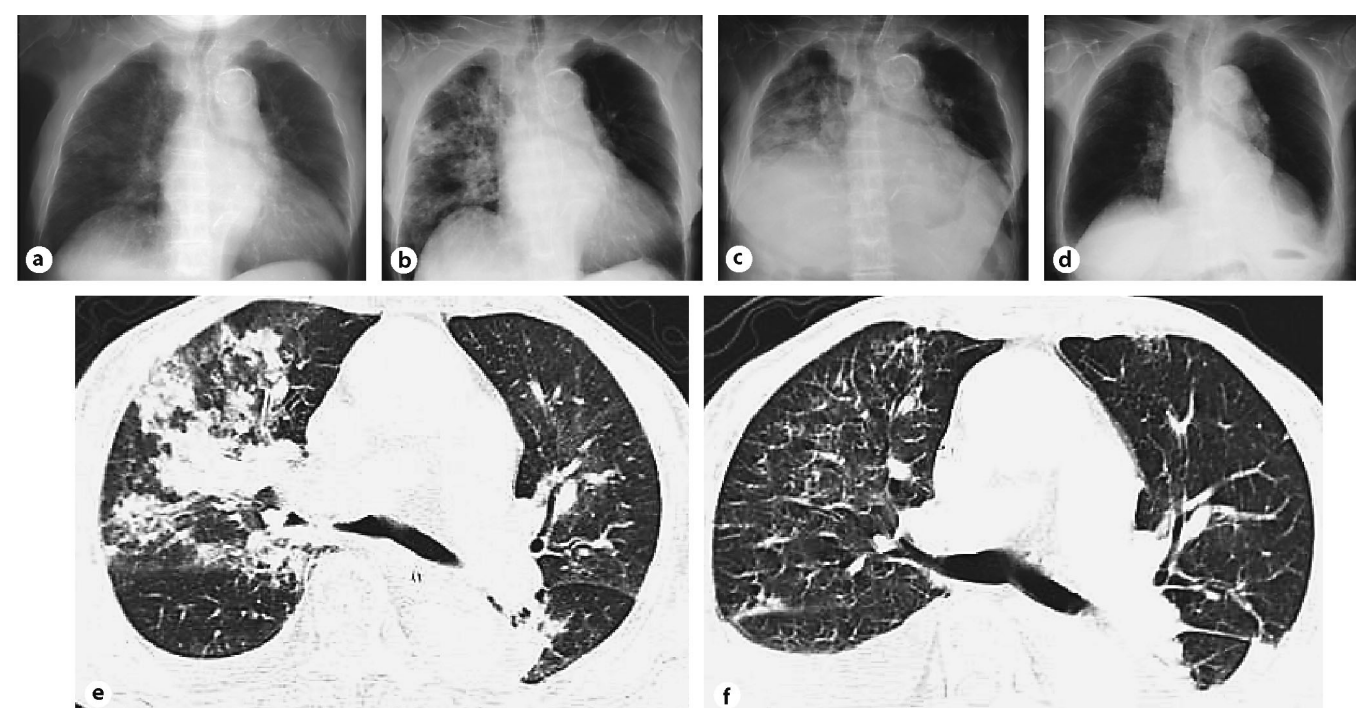

e
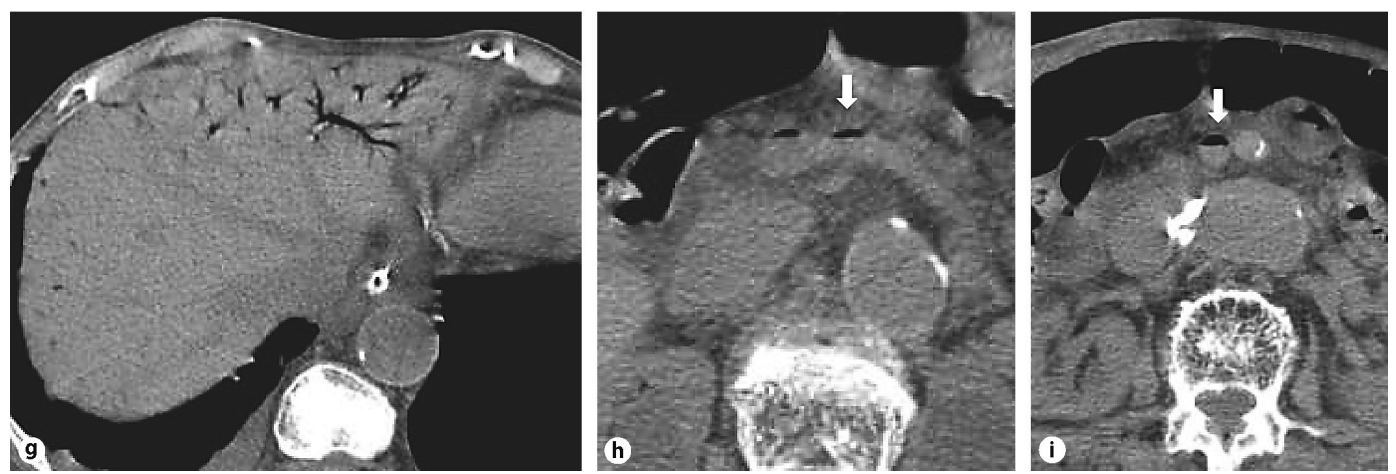


\section{References}

1 Muth CM, Shank ES: Gas embolism. N Engl J Med 2000;342:476-482.

2 Durant TM, Long J, Oppenheimer MJ: Pulmonary (venous) air embolism. Am Heart J 1947;33:269-281.

-3 Barak M, Katz Y: Microbubbles. Chest 2005;128:2918-2932.

$\checkmark 4$ Tommasino C, Rizzardi R, Beretta L, Venturino M, Piccoli S: Cerebral ischemia after venous air embolism in the absence of intracardiac defects. J Neurosurg Anesthesiol 1996;8:30-34.

5 Gronert GA, Messick JM Jr, Cucchiara RF, Michenfelder JD: Paradoxical air embolism from a patent foramen ovale. Anesthesiology 1979;50:548-549.

6 Lynch JJ, Schuchard GH, Gross CM, Wann LS: Prevalence of right-to-left atrial shunting in a healthy population: detection by Valsalva maneuver contrast echocardiography. Am J Cardiol 1984;53:1478-1480.

7 Moon RE: Gas embolism; in Oriani G, Marroni A, Wattel F (eds): Handbook on Hyperbaric Medicine. Milan, Springer, 1996, pp 229-248.

8 Wolfe JN, Evans WA: Gas in the portal veins of the liver in infants; a roentgenographic demonstration with postmortem anatomical correlation. Am J Roentgenol Radium Ther Nucl Med 1955;74:486-488.

-9 Susman N, Senturia HR: Gas embolization of the portal venous system. Am J Roentgenol Radium Ther Nucl Med 1960;83:847-850.

10 Liebman PR, Patten MT, Manny J, Benfield JR, Hechtman HB: Hepatic-portal venous gas in adults: etiology, pathophysiology and clinical significance. Ann Surg 1978;187:281-287.

11 Mallens WM, Schepers-Bok R, Nicolai JJ, Jacobs FA, Heyerman HG: Portal and systemic venous gas in a patient with cystic fibrosis: CT findings. AJR Am J Roentgenol 1995;165:338-339.

12 Root B, Levy MN, Pollack S, Lubert M, Pathak K: Gas embolism death after laparoscopy delayed by 'trapping' in portal circulation. Anesth Analg 1978;57:232-237.

-13 Kriegshauser JS, Reading CC, King BF, Welch TJ: Combined systemic and portal venous gas: sonographic and CT detection in two cases. AJR Am J Roentgenol 1990;154:1219-1221.

14 Butler BD, Morris WP: Transesophageal echocardiographic study of decompression-induced venous gas emboli. Undersea Hyperb Med 1995;22:117128.

15 Malin SW, Buthani VK, Ritchie WW, Hall ML, Paul D: Echogenic intravascular and hepatic microbubbles associated with necrotizing enterocolitis. J Pediatr 1983;103:637-640.

16 Wiot JF, Felson B: Gas in the portal venous system. Am J Roentgenol Radium Ther Nucl Med 1961;86:920-929.

17 Jones B: Massive gas embolism in E. coli septicemia. Gastrointest Radiol 1981;6:161-163.

-18 Etsuda H, Miyamoto A, Hakamata N, Fukuda M, Yamauchi Y, Akita T: Right coronary air embolism secondary to bowel infarction: a case report. J Cardiol 2004;43:141-145.

19 Kinoshita H, Shinozaki M: Clinical features and management of hepatic portal venous gas: four case reports and cumulative review of the literature. Arch Surg 2001;136:1410-1414.

20 Yoshida M, Mitsuo M, Kutsumi H, Fujita T: A successfully treated case of multiple liver abscesses accompanied by portal venous gas. Am J Gastroenterol 1996;91:2423-2425.

-21 Gluecker T, Capasso P, Schnyder P, Gudinchet F, Schaller MD, Revelly JP, Chiolero R, Vock P, Wicky S: Clinical and radiologic features of pulmonary edema. Radiographics 1999;19:1507-1531.

22 Voorhies RM, Fraser RA, Van Poznak A: Prevention of air embolism with positive end expiratory pressure. Neurosurgery 1983;12:503-506.

23 Jaffe RA, Pinto FJ, Schnittger I, Siegel LC, Wranne B, Brock-Utne JG: Aspects of mechanical ventilation affecting interatrial shunt flow during general anesthesia. Anesth Analg 1992;75:484-488. 
24 Giebler R, Kollenberg B, Pohlen G, Peters J: Effect of positive end-expiratory pressure on the incidence of venous air embolism and on the cardiovascular response to the sitting position during neurosurgery. Br J Anaesth 1998;80:30-35. 